\title{
Estilo de vida asociado a autoestima y variables demográficas en estudiantes de enfermería
}

\author{
Lifestyle associated to self-esteem and demographics variables in nursing students
}

Jack Roberto Silva Fhon ${ }^{*}$, Melanie Ayala Caire², José Aparecido Valério Mendonça Júnior ${ }^{3}$, Rosalina Aparecida

Partezani Rodrígues ${ }^{4}$

\begin{abstract}
RESUMEN
Objetivo: Verificar la asociación entre el estilo de vida con autoestima y variables demográficas de los estudiantes de enfermería de una universidad privada. Material y Métodos: Estudio cuantitativo, descriptivo y transversal con 183 estudiantes del 3 al 8 ciclo de enfermería. Fue utilizado el Instrumento de perfil demográfico, Inventario de autoestima - adultos (Forma C) e Instrumento de estilos de vida. Para el análisis de los datos fue utilizado la estadística descriptiva, Prueba de correlación de Pearson y Chi cuadrado ( $\mathrm{p}<0.05)$. Resultados: El 79.2\% era mujer, el 84.2\% casado, el 69.9\% trabajaba. El 41.5\% indica tener una autoestima medio alto y el 53.6\% un estilo de vida saludable. No se encontró correlación entre estilos de vida y edad del participante y se verificó asociación entre los niveles del estilo de vida y los niveles de autoestima. Conclusiones: Existe asociación entre el estilo de vida y autoestima en el estudiante de enfermería.
\end{abstract}

Palabras clave: Estudiantes de enfermería, estilo de vida, autoimagen, Enfermería.

\begin{abstract}
Objective: To verify the association between lifestyle with self-esteem and demographic variables to the nursing students at a private university. Methods: Quantitative, descriptive and cross-sectional research with 183 students from second to fourth year of nursing. We used the demographic instrument, Self-Esteem Inventory - Adults (Form C) and Lifestyle instrument. For data analysis we used descriptive statistic, Pearson correlation and Chi square test $(\mathrm{p}<0.05)$. Results: $79.2 \%$ were women, $84.2 \%$ were married, $69.9 \%$ worked. $41.5 \%$ reported to have a medium high self-esteem and $53.6 \%$ had healthy lifestyle. We didn't find correlation between life styles and the age to de student. We verified the association between the lifestyle levels and self-esteem levels. Conclusion: We found there is association between lifestyle and self-esteem in the nursing student.
\end{abstract}

Keywords: Students nursing, Life style, Self-concept, Nursing.

\footnotetext{
${ }^{1}$ Magíster en Ciencias, Escuela de Enfermería de Ribeirão Preto - Universidad de São Paulo, Brasil.

${ }^{2}$ Enfermera, Escuela Académico Profesional de Enfermería - Universidad Privada Norbert Wiener, Perú.

${ }^{3}$ Pedagogo, Red Municipal de Educación de Batatais, Brasil.

${ }^{4}$ Doctora en enfermería, Escuela de Enfermería de Ribeirão Preto - Universidad de São Paulo, Brasil.
} 


\section{INTRODUCCIÓN}

Los estilos de vida son el conjunto de comportamientos y hábitos que tiene cada persona en determinados aspectos de la vida, los cuales influyen directamente en la salud o en la enfermedad. (Maya, 2001).

La Organización Mundial de la Salud (OMS) la define como "el conjunto de patrones de comportamiento identificable y relativamente estable en el individuo o en una sociedad determinada; resulta de las interacciones entre las características individuales, la interacción social y las condiciones de vida socioeconómica y ambiental en que viven las personas” (OMS, 1994). Además, puede estar determinado por aspectos individuales como el biológico, genético, psicológico y conductual; aspectos sociodemográficos y culturales que son influencias del contexto social, económico y del medio ambiente (De la Cruz y Pino, 2010).

De esta manera, el estilo de vida puede llegar a ser saludable siguiendo una conducta positiva logrando un adecuado mantenimiento de la salud, siendo determinado por las elecciones que se hace a lo largo de la vida, de acuerdo a sus conocimientos y su capacidad de autocuidado (Cockerham, 2007). El modelo de promoción de la salud refiere que existen determinantes biopsicosociales que motivan al individuo a adoptar conductas positivas y/o negativas, entre estas la autoestima, como parte de la esfera psicológica del ser humano (Aristizábal et al., 2011).

La autoestima es confiar en las propias potencialidades, es definida por dos aspectos fundamentales: a) sentido de eficacia personal o autoeficacia, que es la confianza en el funcionamiento de la mente, en la capacidad de pensar en los procesos por los cuales se juzga, se elige y se decide (Almuneda, 2003) y b) sentido de mérito personal o autodignidad, es la seguridad del valor propio, una actitud afirmativa hacia el derecho de vivir y de ser feliz. Es la comodidad al expresar apropiadamente los pensamientos, deseos y necesidades; sentir que la alegría es el derecho natural. (Miranda, 2005).

Para fines del presente estudio, fue utilizado el referencial teórico de Coopersmith (1995), que afirma que la autoestima puede presentarse en tres niveles: alta, media y/o baja, evidenciándose porque las personas experimentan las mismas situaciones en forma notablemente diferente.

La autoestima es considerada como un factor protector para conductas dañinas para la salud (Álvarez et al., 2010), del mismo modo, presentar una alta autoestima es un predictor de bienestar general entre los estudiantes (Gómez-Bustamante y Cogollo, 2010).

El estilo de vida y la autoestima, en los estudiantes de enfermería, son de vital importancia para el desarrollo personal e intelectual. En esta etapa se enfrentan a las experiencias teóricas y prácticas, lo que genera un impacto influenciando en su rendimiento académico. Los estudiantes tienen que lidiar con una vida universitaria agitada, donde la adquisición de conocimientos es realizada en la universidad como en las diferentes sedes de práctica, además del trabajo para su manutención y las responsabilidades en el hogar, generando cambios en el autocuidado personal.

De esta manera, por medio de la revisión de la literatura, el estudio tuvo como objetivo principal verificar la asociación entre el estilo de vida con autoestima y las variables demográficas de los estudiantes de enfermería de una universidad privada.

\section{MATERIALES Y MÉTODOS}

El estudio fue cuantitativo, descriptivo de corte transversal. Los participantes fueron estudiantes de enfermería de una universidad privada de la ciudad de Lima. La recolecta de las informaciones fue realizada en el período de agosto - noviembre del 2013. Los criterios de inclusión fueron: ambos sexos, estar matriculados en el ciclo académico 2013 - I y estudiantes del 3er al 8vo ciclo.

La población de estudio estuvo constituida por 349 estudiantes, para la obtención de la muestra fue utilizada la fórmula para población conocida con un nivel de confiabilidad del 95\%, siendo constituida por 183 estudiantes. Para la obtención de la muestra final fue realizado un muestreo estratificado, para conocer la cantidad de estudiantes de acuerdo a cada ciclo académico.

Fueron utilizados el instrumento de Caracterización del estudiante donde se conoció el sexo (masculino, femenino), edad en años, estado civil (soltero, casado, conviviente), ciclo académico, horario de clases (mañana, noche) y situación laboral (sí, no).

El Inventario de Autoestima - Adultos (Forma C) de Stanley Coopersmith adaptada y estandarizada consta de 25 ítems, distribuidos en tres áreas, hacia sí mismo (13 preguntas); área social - pares (6 preguntas) y área familiar (6 preguntas). El puntaje máximo es de 100 puntos y no existe escalas de mentiras que invaliden la prueba. Los puntajes se obtienen sumando el número de ítems respondido en forma correcta, la puntuación varía de 0 a 100 puntos, caracterizándose como nivel bajo aquellos con puntuación de 0 a 24 puntos; nivel 
medio bajo de 25 a 49; nivel medio alto de 50 a 74 y nivel alto de 75 a 100 (Matos, 1999).

Para el estilo de vida, fue elaborado un cuestionario con escala numérica tipo Likert, consta de 38 reactivos. Presenta cuatro criterios de respuesta, para preguntas positivas (nunca $=1$; a veces $=2$; frecuentemente $=3$; siempre $=4$ ) y para preguntas negativas (nunca $=4$; a veces=3; frecuentemente $=2$; siempre=1). Fueron realizadas preguntas sobre actividad física (4 preguntas), actividades recreativas (5 preguntas), hábitos alimenticios (12 preguntas), consumo de sustancias nocivas (4 preguntas), sueño (6 preguntas) y responsabilidad con la salud (7 preguntas).

El instrumento fue validado a través de juicio de expertos y posteriormente se sometió a prueba binomial. Se realizó una prueba piloto para determinación de la confiabilidad del instrumento mediante la prueba de Alfa de Crombach (0.79), lo cual indica una fuerte confiablidad.

La puntuación de la escala varía de 38 a 152 puntos, siendo caracterizados como estilos de vida no saludables entre 38 a 94 puntos; estilos de vida medianamente saludables entre 95 a 109 puntos y estilos de vida saludables entre 110 a 152 puntos (Aiken 2003).
Para el análisis de los datos fue realizada una tabulación de doble entrada, en el programa Microsoft Excel ${ }^{\circledR}$, para observar los posibles errores e ir al instrumento original realizando la corrección respectiva, posteriormente fueron transportados los datos al programa estadístico Statistical Package for the Social Sciences - SPSS v. 22.0. Para las variables cuantitativas fue utilizada la estadística descriptiva, con uso de frecuencias, medida de tendencia central (media) y de dispersión (desviación estándar). Asimismo, fue utilizado el análisis de correlación de Pearson entre la puntuación total del instrumento de estilo de vida, con la edad del entrevistado.

Para el análisis de asociación, fueron utilizadas las categorías del estilo de vida y las variables demográficas sexo (masculino y femenino), estado civil sin compañero (soltero) y con compañero (casado y conviviente), trabajo (sí y no), turno de clases (mañana y noche) y las categorías de autoestima realizándose la prueba Chi cuadrado. Para todos los análisis se tuvo un nivel de significancia de $\mathrm{p}<0.05$.

El estudio fue aprobado por el comité de investigación de grados y títulos de la Universidad Privada Norbert Wiener, además se garantizó los principios éticos del Tratado de Helsinki, lo que aseguró el anonimato del entrevistado.

\section{RESULTADOS}

Tabla 1

Perfil demográfico del estudiante de pregrado en enfermería. Lima

\begin{tabular}{|c|c|c|c|c|}
\hline Variables & Media (=DE) & Variación & $\mathrm{n}$ & $\%$ \\
\hline \multicolumn{5}{|l|}{ Sexo } \\
\hline Masculino & & & 38 & 20.8 \\
\hline Femenino & & & 145 & 79.2 \\
\hline Grupo etario & $25.57(4.490)$ & {$[18-40]$} & & \\
\hline $18-23$ & & & 61 & 33.3 \\
\hline $24-29$ & & & 88 & 48.1 \\
\hline $30-34$ & & & 25 & 13.7 \\
\hline $35-39$ & & & 8 & 4.4 \\
\hline Mayor de 40 & & & 1 & 0.5 \\
\hline \multicolumn{5}{|l|}{ Estado civil } \\
\hline Soltero & & & 154 & 84.2 \\
\hline Casado & & & 19 & 10.4 \\
\hline Conviviente & & & 10 & 5.5 \\
\hline \multicolumn{5}{|c|}{ Trabaja el estudiante } \\
\hline Sí & & & 128 & 69.9 \\
\hline No & & & 55 & 30.1 \\
\hline \multicolumn{5}{|c|}{ Horario de clases } \\
\hline Mañana & & & 92 & 50.3 \\
\hline Noche & & & 91 & 49.7 \\
\hline
\end{tabular}


Estilo de vida asociado a autoestima y variables demográficas en estudiantes de enfermería

En la Tabla 1 se observa la caracterización de los participantes, se observó predominio del sexo femenino (79.2\%), entre edades de 24 y 29 años
(48.1\%), solteros (84.2\%), trabajan (69.9\%) y que estudian en el turno mañana (50.3\%).

Tabla 2

Caracterización de autoestima y estilos de vida de los estudiantes de pregrado en enfermería. Lima

\begin{tabular}{lllll}
\hline Variables & Media $(=\mathrm{DE})$ & Variación & $\mathrm{n}$ & $\%$ \\
\hline Autoestima & $13.03(5.51)$ & {$[3-25]$} & & \\
Alto & & & 20 & 10.9 \\
Medio alto & & 76 & 41.5 \\
Medio bajo & & 58 & 31.7 \\
Bajo & & 29 & 15.8 \\
Estilo de vida & $101.58(9.74)$ & {$[78-130]$} & & \\
Saludable & & & 98 & 53.6 \\
Medianamente & & & 41 & 22.4 \\
saludable & & & 44 & 24.0 \\
No saludable & & & \\
\hline
\end{tabular}

Por otro lado, la Tabla 2 muestra la evaluación de la autoestima por medio del instrumento de Coopersmith, el $41.5 \%$ de los participantes refirieron tener una autoestima medio alto y $31.7 \%$ tener autoestima medio bajo. A la determinación del estilo de vida, el 53.6\% indicó un estilo de vida saludable y $24 \%$ refirió un estilo de vida no saludable.

\section{Tabla 3}

Asociación entre autoestima y variables sociodemográficas y estilo de vida en estudiantes de pregrado en enfermería. Lima, 2014

\begin{tabular}{|c|c|c|c|c|}
\hline & Estilo de vida & Medianamente & No Saludable & \\
\hline Variables & $\mathrm{n}(\%)$ & $\mathrm{n}(\%)$ & $\mathrm{n}(\%)$ & $p$-valor \\
\hline \multicolumn{5}{|l|}{ Sexo } \\
\hline Masculino & 24 (63.2) & $6(15.8)$ & $8(21.1)$ & 0.379 \\
\hline Femenino & $74(51.0)$ & $34(24.1)$ & $36(24.8)$ & \\
\hline \multicolumn{5}{|l|}{ Estado civil } \\
\hline Con compañero & $17(58.6)$ & $4(13.8)$ & $8(27.6)$ & 0.476 \\
\hline Sin compañero & $81(52.6)$ & $37(24.0)$ & $36(23.4)$ & \\
\hline \multicolumn{5}{|l|}{ Trabajo } \\
\hline Si & $65(50.8)$ & 27 (21.1) & $36(28.1)$ & 0.143 \\
\hline No & & $33(60.0)$ & $14(25.5)$ & $8(14.5)$ \\
\hline \multicolumn{5}{|l|}{ Turno de clases } \\
\hline Mañana & $52(56.5)$ & $22(23.9)$ & $18(19.6)$ & 0.361 \\
\hline Noche & $46(50.5)$ & $19(20.9)$ & $26(28.6)$ & \\
\hline \multicolumn{5}{|l|}{ Autoestima } \\
\hline Alto & $18(90.0)$ & $2(10.0)$ & - & $0.000^{*}$ \\
\hline Medio alto & $49(64.5)$ & $17(22.4)$ & $10(13.2)$ & \\
\hline Medio bajo & $19(32.8)$ & 17 (29.3) & $22(37.9)$ & \\
\hline Bajo & $12(41.4)$ & $5(17.2)$ & $12(41.4)$ & \\
\hline
\end{tabular}


A la correlación de Pearson entre la puntuación total del instrumento del estilo de vida y edad del participante, según muestra la Tabla 3, se encontró un $r=-0.018$ y $p=0.805$. Lo que indica una correlación negativa entre ambas variables sin significancia estadística.

Se verificó asociación entre los niveles del estilo de vida con los niveles de autoestima ( $\mathrm{p}>0.05)$.

\section{DISCUSIÓN}

La enfermería es una profesión universitaria con una formación integral basada en principios científicos, humanísticos y tecnológicos para brindar un cuidado integral y de alta calidad al individuo, la familia y la comunidad en todas las etapas del ciclo vital (Rivas, 2010).

Los resultados obtenidos, en el presente estudio, permiten identificar que la mayoría de los estudiantes son mujeres. Estudios sobre el tema encuentran porcentajes entre 85\% (Cruz, 2012) y 85.3\% (Mescua, 2010) de mujeres que estudian enfermería. Asimismo, se encontró una mayor proporción de los estudiantes se encuentran en el grupo etario comprendido entre 24 y 29 años. Un estudio peruano encontró que 49.3\% de los estudiantes de la facultad de enfermería tenían edades comprendidas entre 23 y 28 años, señalándose que son adultos jóvenes en plena formación académica y personal (Mescua, 2010).

En relación al estado civil, los resultados indican que la mayoría de estudiantes son solteros, datos similares fueron encontrados por Ponte (2010), donde el 91.25\% de estudiantes eran solteros. De acuerdo al trabajo el 69.9\% de estudiantes mencionaron que desempeñan alguna actividad laboral, datos similares se encontraron en un estudio sobre jóvenes universitarios, donde señalaban que el 61.8\% de estudiantes trabajaban, por diferentes necesidades; algunos para satisfacer sus necesidades, para sustentar sus estudios o en búsqueda de su independencia personal; siendo el trabajo y el estudio al mismo tiempo un factor para desencadenar situaciones de estrés, bajo rendimiento académico y afecciones de salud (Centro de estudios de opinión ciudadana, 2010).

La autoestima es la suma de la confianza y el respeto que debemos sentir por nosotros mismos, refleja el juicio de valor individual para enfrentar los desafíos. En el presente estudio se verificó que predominó el nivel de autoestima medio alto. Un estudio realizado con 100 estudiantes de enfermería, el 54\% tuvo autoestima media, indicando que la mayoría de alumnos podría no valorizar su propio potencial, por lo que existirían brechas en el cumplimiento de su labor, siendo importante estimular los logros de los estudiantes, a fin de que ellos reconozcan su capacidad en las diferentes actividades académicas (Moreno, 2010).

Similar resultado se encontró en otros estudios, donde el 45.59\% (Gamarra et al., 2010) y el 40\% (Mescua, 2010) de los estudiantes de enfermería presentaron niveles de autoestima medio, lo que podría indicar que la mayoría de estudiantes son personas a quienes se les hace difícil tomar decisiones, enfrentar retos con facilidad, miedo al fracaso y se dejan llevar por lo demás; sin embargo, existen conductas positivas que requieren ser reforzadas.

La personalidad del individuo es una unidad organizada e integrada, constituida por muchos elementos que actúan conjuntamente, siendo la autoestima uno de los componentes importantes para su desarrollo (Padillo, 1998). Todas las personas, en especial el enfermero, necesitan tener una autoestima alta que le permita desarrollar habilidades, generar conductas asertivas con el fin de brindar un cuidado integral y de alta calidad para el bienestar de sí mismo y del usuario.

La salud está integrada por factores o determinantes, siendo uno de los componentes el estilo de vida, relacionado a los hábitos personales y grupo de la alimentación, tipo de actividad física, adicciones, conductas peligrosas entre otras que pueden limitar el desarrollo humano o reducir las oportunidades de salud en el individuo (Villar, 2011)

Los estudiantes de enfermería, como futuros educadores, están encargados de la promoción de la salud del individuo, familia y comunidad, por ende, deben ser los primeros en tener conductas saludables. Las universidades tienen el compromiso formativo integral, debido que trasciende la formación académica y social, por lo que deben promocionar un escenario saludable.

Los resultados encontrados apuntan a que la mayoría de los entrevistados presentaron tener un estilo de vida saludable, datos similares fueron encontrados en otros estudios, variando entre $52.2 \%$ (Cid et al., 2006) y 60\% (Ponte, 2010). Sin embargo, los datos difieren del estudio de Gamarra et al., (2010) donde el $54.4 \%$ practica estilos de vida medianamente saludables; por lo que existen ciertas conductas en los alumnos que los conducen a desajustes biológicos, psicológicos y sociales en contra de su salud y en contra del mejoramiento de su calidad de vida y su desarrollo personal.

Asimismo, un estudio con una población variada de estudiantes del área de salud, se observó estilos 
de vida poco saludable (Arguello et al., 2009), identificándose que la información no es suficiente para adoptar comportamientos saludables, así el hecho de recibir formación profesional en áreas de la salud no es suficiente para producir cambios en los hábitos de vida.

El estilo de vida puede verse influenciado por factores internos y externos, resultados señalan que hubo asociación entre la autoestima y estilos de vida del estudiante de enfermería. Del mismo modo, un estudio sobre el tema encontró una relación significativa inversa entre estas dos variables, por lo que afirmó que cuanto mayor es la percepción del comportamiento como adecuado, menor es el riesgo de conductas inapropiadas, menos consumo de alimentos insanos y mayor inclinación por la actividad física (Gamarra et al., 2010). Bezzolo (2012) asociando las dos variables encontró relación positiva entre los niveles de autoestima y estilo de vida, y que dicha correlación presenta diferencias significativas al compararla por sexos.

La autoestima es importante para explicar el estilo de vida como promotor de la salud, por lo que se menciona que presentar niveles más altos de autoestima está prácticamente asociada a puntuaciones más altos de estilos de vida (Cid et al., 2006).
Se concluye el predomino del nivel de autoestima medio alto y estilo de vida saludable. Asimismo, se observó significancia estadística entre los niveles de autoestima y estilos de vida.

Es importante que las universidades públicas y privadas mantengan el compromiso de promover la educación para la salud, como una estrategia que favorezca a los estudiantes, contribuyendo en su formación personal y profesional.

\section{Declaración de financiamiento y de conflicto de intereses:}

El estudio fue financiado por los autores, quienes declaran no tener algún tipo de conflicto de interés en la investigación realizada.

\section{Correspondencia:}

Jack Roberto Silva Fhon.

Rua Machado de Assis 971 Bairro Vila Tibério, Ribeirão Preto, São Paulo. Brasil. CEP 14050-490. e-mail: beto_fhon@hotmail.com

\section{REFERENCIAS BIBLIOGRÁFICAS}

Aiken. L. R. (2003). Análisis de reactivos y estandarización de pruebas. En: Tests psicológicos y evaluación. 11 ed México: Pearson educación. p. 544.

Almuneda, O. A. (2003). Factor individual: Autoestima. Curso de enfermería de empresa. Recuperado el 14 de mayo de 2014, de http://www.enfermeriasalamanca.com/ TRABAJOS_SALUD_LABORAL/SEGURIDAD/ AUTOESTIMA.PDF

Álvarez, A. A., Alonso, C. M. M., Guidorizzi, Z. A. C. (2010). Consumo de alcohol y autoestima en adolescentes. Rev. Latino-Am. Enfermagem 18(Spec), 634-40

Arguello, M., Bautista, Y., Carvajal, J., de Castro, K., Díaz, D., Escobar, M. et al. (2009). Estilos de vida en estudiantes del área de la salud de Bucaramanga. Rev de Psicología 1(2), 27-41.

Aristizábal, H. G. P., Blanco, B. D. M., Sánchez, R. A., Ostiguín, M. R. M. (2011). El modelo de promoción de la salud de Nola Pender. Una reflexión en torno a su comprensión. Enfermería universitaria 8(4), 16-23.

Bezzolo, T. G. (2012). Autoestima y estilo de vida en estudiantes universitarios de la carrera de ciencias de la comunicación, Chiclayo 2012. Tesis, Universidad Católica Santo Toribio de Mogrovejo, Chiclayo, Perú.

Centro de Estudios de Opinión Ciudadana. (2010). Actividad laboral de los jóvenes universitarios. Chile: Universidad de Talca. Recuperado el 18 de junio del 2014, de
http://www.ceoc.cl/pdf/Estudios_Opinion/2010/0210Actividad_Laboral_Universitarios.pdf

Cid, H. P., Merino, E. J. M., Stiepovich, B. J. (2006). Factores Biológicos y Psicosociales predictores del estilo de vida promotor de la salud. Rev. Med Chile 134(12), 1491-99.

Cockerham, W. C. (2007). New directions in health lifestyle research. Int J Public Health 52(6), 327-8.

Coopersmith, S. (1995). The Antecedents of Self Esteem Consulting. $1^{\circ}$ ed. USA: Psychologist Press.

Cruz, N. F., Quinones, U. A. (2012). Autoestima y rendimiento académico en estudiantes de enfermería de Poza Rica, Veracruz, México. Uni-pluri/versidad 12(1), 25-35.

De la Cruz, S. E., Pino, O. J. (2010). Estilo de vida relacionado con la salud. Arch Murcia. 20(supl), 4-6.

Gamarra, S. M., Rivera, T. H., Alcalde, G. M., Cabellos, V. D. (2010). Estilo de vida, autoestima y apoyo social en estudiantes de enfermería. Rev. Salud Lima 2(2), 73-9.

Gómez, E. M., Cogollo, Z. (2010). Factores predictores relacionados con el bienestar general en adolescentes estudiantes de Cartagena, Colombia. Rev. Salud pública 12(1), $61-70$

Maya, S. L. H. (2001). Los estilos de vida saludables: componente de la calidad de vida. Funlibre. Recuperado 
el 16 de Setiembre 2014, de http://www.funlibre.org/ documentos/lemaya1.htm

Matos, A. R. (1999). Manual: Inventario de autoestima adultos Forma C, Stanlet Coopersmith.

Mescua, Ñ. G. D. (2010). Relación entre el nivel de autoestima y el tipo de familia procedente de los estudiantes de enfermería de la Universidad Nacional Mayor de San Marcos, 2009 Tesis. Facultad de Medicina Humana, Universidad Mayor de San Marcos, Lima, Perú.

Miranda, C. (2005). La autoestima profesional: una competencia mediadora para la innovación en las prácticas pedagógicas. REICE 3(1). 858-873.

Moreno, E. L., Torres, V. T. (2010). Autoestima y su relación con el rendimiento académico de los estudiantes del $4^{\circ}$ al $8^{\circ}$ ciclo de la EAPE. Tesis. Escuela de Académico Profesional de Enfermería, Universidad Privada Norbert Wiener, Lima, Perú.
Organización Mundial de la Salud (1994). Las Condiciones de Salud en las Américas. Washington 35, 22-6.

Padillo, C. (1998). Autoestima para estudiantes. $1^{\circ} \mathrm{ed}$. México: Iboamericana.

Ponte, A. M. (2010). Estilos de vida de los estudiantes de enfermería, según año de estudio. Tesis. Facultad de Medicina Humana, Universidad Mayor de San Marcos, Lima, Perú.

Rivas, D. L. H. (2010). Relación entre el desempeño docente y el logro de objetivos educacionales de estudiantes de la E.A.P. de Enfermería de la UNMSM, 2010. Tesis. Facultad de Educación, Universidad Nacional Mayor de San Marcos, Lima, Perú.

Villar, A. M. (2011). Factores determinantes de la salud: Importancia de la prevención. Acta Med Per 28(4), 237241. 